\title{
PROBLEMAS DE COBERTURA EN LA ENCUESTA TELEFÓNICA*
}

\author{
Vidal Díaz de Rada \\ Universidad Pública de Navarra \\ E-mail: vidal@unavarra.es
}

\begin{abstract}
RESUMEN
El objetivo de este trabajo es analizar uno de los principales problemas de las encuestas telefónicas, referido concretamente al procedimiento de estimación y conocimiento de las personas que no pueden ser entrevistadas telefónicamente por no disponer de teléfono; definido como error de cobertura. Pese a la implantación "masiva» del teléfono en la mayor parte de los hogares de los países desarrollados, no toda la población dispone en su hogar de este medio de comunicación, existiendo colectivos que todavía carecen de este equipamiento. El gran problema que produce la no cobertura es la pérdida de representación que se comete al obviar un determinado tipo de personas, de modo que este descenso en la representatividad se traduce en una pérdida de capacidad de inferencia de la muestra, produciendo un sesgo en el proceso de inferencia. La cuantificación de estos colectivos y el conocimiento de sus características son los objetivos principales de este trabajo, en la medida que nos permiten conocer la magnitud del error de cobertura en las encuestas telefónicas realizadas en nuestro país.
\end{abstract}

Las encuestas telefónicas comienzan a utilizarse en Estados Unidos en los años treinta (Massey, 1988a: 3), aunque su gran desarrollo no se produce hasta la segunda mitad de este siglo con la creación y desarrollo de sistemas de entrevistas centralizadas (Nicholls, 1988: 397). En la actualidad, la entrevista telefó-

* El autor desea agradecer la ayuda concedida por el Centro de Investigaciones Sociológicas para la explotación del estudio Los comportamientos económicos de los españoles: ahorro y consumo. 
nica es el procedimiento de recogida de datos más utilizado en la investigación social que se realiza en la mayor parte de los países europeos y en Estados Unidos (Groves et al., 1988: 3; Czaja y Blair, 1996: 38). Respecto a este último, Wert (1996b) estima que la entrevista telefónica es utilizada en más del 70 por 100 de las investigaciones.

\section{TABLA 1}

\section{Entrevistas por teléfono en paises europeos \\ (En porcentajes)}

\begin{tabular}{|c|c|}
\hline Suiza ...... & 63 \\
\hline Suecia ............... & 58 \\
\hline Dinamarca .................. & 53 \\
\hline 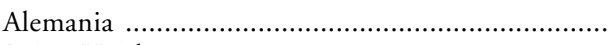 & 44 \\
\hline 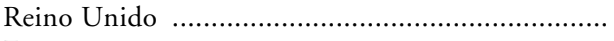 & 37 \\
\hline 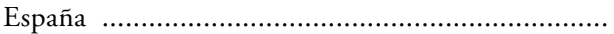 & 29 \\
\hline
\end{tabular}

Fuente: Blyth (1998). Citado en Fernández Prados (1999: 32).

A la hora de explicar la rapidez de este desarrollo, Lavrakas señala la importancia de una serie de factores físicos y sociales (1993: 8-18). Entre los primeros destaca la implantación masiva del teléfono en los hogares, el desarrollo de una teoría específica sobre este procedimiento de recogida de información, los avances del teléfono y el desarrollo de la tecnología de entrevistas por teléfono y, por último, la integración de los procesos de recogida y tratamiento de la información mediante los sistemas de entrevistas centralizadas. En relación a los factores sociales, destacar la menor invasión de la privacidad que supone contestar a una encuesta por teléfono, más sinceridad en temáticas delicadas, el hecho que el teléfono siempre es contestado porque no se conoce a priori la persona que llama y la complejidad de la sociedad actual, que requiere información actualizada en cortos períodos de tiempo. A estos factores hay que añadir el progresivo descenso en la tasa de respuesta de las encuestas personales, como han señalado - entre otrosBethlehem y Kersen (1981), Steeh (1981), Goyder (1985 y 1987) y Sugiyama (1992).

El objetivo de este trabajo es analizar uno de los principales problemas de las encuestas telefónicas, referido concretamente al procedimiento de estimación y conocimiento de las personas que no pueden ser entrevistadas telefónicamente por no disponer de teléfono; definido como error de cobertura. Pese a la implantación «masiva» del teléfono en la mayor parte de los hogares de los países desarrollados, no toda la población dispone en su hogar de este medio de comunicación, existiendo colectivos que todavía carecen de este equipa- 
miento. La cuantificación de estos colectivos y el conocimiento de sus características son los objetivos principales de este trabajo, en la medida que nos permiten conocer la magnitud del «error de cobertura» en las encuestas telefónicas realizadas en nuestro país.

Al margen de otros aspectos como la calidad de la información recogida y la rapidez en la recolección y procesamiento de la información, la elección de la encuesta telefónica va a depender de un "aspecto técnico» como es el número de hogares sin teléfono en la población objeto de estudio. Sin embargo, en algunas investigaciones la necesidad de recoger rápidamente la información adquiere una importancia crucial a la hora de elegir el procedimiento de recogida de información, y ésta es la principal razón por la que se utiliza la encuesta telefónica.

Un ámbito de estudio donde la rapidez de recogida y procesamiento es fundamental es el estudio de la conducta electoral. El hecho que el comportamiento electoral pueda experimentar rápidas variaciones por la intensidad de la campaña electoral, por la influencia de factores puntuales como escándalos financieros o políticos, etc., aconseja realizar la recogida de información en un breve período de tiempo con el fin de "controlar» todos los factores «externos». La mayor parte de los sondeos electorales mediante encuestas telefónicas realizan la recogida de datos en menos de nueve días, "velocidad» que es muy difícil de conseguir mediante una encuesta personal, y mucho menos una encuesta postal.

A estos factores hay que añadir que la legislación española limita la publicación de resultados de encuestas electorales una semana antes del día de los comicios, de modo que la recogida de información debe realizarse muy rápidamente con el fin que esté disponible diez días antes de la concurrencia electoral. Para publicar los resultados una semana antes del día 12 de marzo de 2000 - por considerar las últimas elecciones-, la mayor parte de los sondeos electorales comenzaron la recogida de datos el 21 de febrero, una semana antes del comienzo «oficial» de la campaña electoral (ver Anexo).

Durante los últimos días de la campaña electoral suele producirse una inflexión significativa de tendencias (Wert, 1996a: 10) que, evidentemente, no queda recogida en las encuestas realizadas. A este hecho se han unido otros argumentos para explicar los fracasos de los sondeos electorales, destacando el escaso tamaño de las muestras; valoración excesiva del recuerdo de voto anterior; poca exigencia en la representatividad muestral según sexo, edad, estudios y ocupación; peculiaridades de nuestro sistema electoral; ocultación del voto y ponderación (no muy acertada) de los resultados brutos; «malestar» hacia ciertos partidos en las elecciones generales de un determinado momento; y el hecho que el que el conocimiento de la predicción puede modificar el comportamiento electoral (entre otros, González, 1998: 191-202; Wert, 1996a: 10; y Trasobares, 2000: 5).

Del párrafo anterior se desprende que los argumentos para explicar los fracasos de los sondeos electorales en diversas concurrencias electorales han sido 
muy variopintos, pero ninguno hace referencia a los problemas de cobertura producidos por las encuestas telefónicas para estimar el voto político.

Si bien al principio del trabajo se ha señalado que éste va a estar referido a cualquier investigación realizada mediante encuestas telefónicas, en el momento presente afecta fundamentalmente a los sondeos electorales, que es el ámbito donde con más profusión se está utilizando la encuesta telefónica. Así, aunque en la tabla 1 se ha señalado que en España la encuesta telefónica se utiliza en una de cada tres investigaciones (29 por 100), si se analiza la temática de cada investigación en los sondeos electorales la utilización de la encuesta telefónica estará cercana al cien por cien; razón por la que nos parece tan importante analizar los problemas de "cobertura telefónica» en el marco de los sondeos electorales. De hecho, de los seis sondeos que se publicaron en prensa el domingo 5 de marzo de 2000, tan sólo uno no fue realizado mediante encuestas telefónicas, como se muestra en el Anexo.

Respecto a la estructura del trabajo, desde nuestro punto de vista, el error de cobertura debe analizarse contextualizado en un ámbito más general dedicado a los errores presentes en la investigación mediante encuestas. El análisis de estos errores permitirá realizar una definición concreta del error de cobertura, y de este error cuando se produce con una técnica específica como son las encuestas telefónicas. El cuarto apartado, dedicado a las variables determinantes del error de cobertura, permitirá conocer los colectivos mejor y peor representados con encuestas telefónicas. El trabajo termina con unas conclusiones donde se recogen una serie de propuestas de reducción del error de cobertura en las encuestas telefónicas.

\section{ERRORES PRESENTES EN LA INVESTIGACIÓN MEDIANTE ENCUESTAS}

Comenzaremos el trabajo ofreciendo una visión general de los errores susceptibles de cometerse en la investigación mediante encuestas. En el cuadro 1 se muestran las fuentes de error, tal y como son especificadas por Benthlehem y Keller (1998: 20). La mayor parte de la literatura especializada distingue entre errores de muestreo, producidos al seleccionar una parte de la población, y errores de no muestreo, mucho más difíciles de detectar y controlar. Dentro de los primeros, el cuadro 1 diferencia entre el error de selección y el error de estimación.

El error de selección se produce por la realización de una mala elección de las personas a entrevistar. El muestreo probabilístico se caracteriza porque se conoce la probabilidad que tiene una persona de ser elegida, utilizando un proceso de selección que respeta tal probabilidad. Ello implica que las personas son seleccionadas por un criterio aleatorio, y no basados en preferencias arbitrarias de los entrevistadores. No obstante, en numerosas ocasiones esta situación no se cumple en las últimas etapas del muestreo, por ejemplo al utilizar el 
muestreo de cuotas, desconsiderando así el criterio fundamental del muestreo probabilístico.

El error de estimación está originado por entrevistar a una parte de la población objeto de estudio, y es un indicador de la precisión de los resultados obtenidos. El nivel de precisión está muy relacionado con el tamaño de la muestra; de modo que a medida que disminuye el tamaño de la muestra se reduce también la precisión de las estimaciones y, por lo tanto, aumenta el error muestral. Una elevada precisión de las estimaciones indica una escasa dispersión de la distribución del estimador en la muestra seleccionada, o, dicho de otro modo, está reflejando una concentración de los valores estimados cerca del valor real.

\section{CUADRO 1}

Errores en la investigación mediante encuesta

Errores de muestreo:

1. Error de selección.

2. Error de estimación.

Errores de no muestreo:

1. Errores de observación:

A) Error por sobrecobertura.

B) Error de medida.

1 Error del entrevistador.

2 Error del entrevistado.

3 Error del instrumento de medida.

4 Error del procedimiento de recogida de información.

5 Error generado por la situación de la entrevista.

C) Error de procesamiento de la información.

2. Errores de no observación:

A) Error por falta de cobertura.

B) Error de no respuesta.

FuENTE: Basado en Benthlehem y Keller (1989: 20).

Los errores de no muestreo son más numerosos que los anteriores, siendo también mucho más difíciles de localizar y controlar (Marton, 1988: 12). Estos errores son divididos en errores de observación y errores de no observación. Dentro de los primeros es posible diferenciar entre: 
A) Errores de sobrecobertura. Se producen por incluir en la muestra elementos que no pertenecen a la población objeto de estudio. En el siguiente apartado se analiza con más detalle este problema.

B) Errores de medida. El error de medida está originado por una inadecuación en la información recogida por el instrumento de medida, y se define como «la diferencia entre el valor observado y el valor real de una determinada unidad» (Marton, 1988: 17). Para Groves (1989), el error de medida puede estar originado por cuatro factores:

1) Errores producidos por una mala actuación de las personas que realizan la recogida de datos, que puede tener su origen en una inadecuada selección, preparación o control de estas personas. A grandes rasgos, podríamos señalar tres grandes tipos de errores producidos por los entrevistadores: cumplimentación de los cuestionarios por parte de los propios entrevistadores, influir de alguna forma en la respuesta de los entrevistados $\mathrm{y}$, por último, una mala recogida de las respuestas de éstos.

2) Los entrevistados son considerados como fuente de error cuando expresan respuestas que no desvelan sus conocimientos o percepciones sobre el tema objeto de estudio. Estos errores pueden producirse por la ausencia de conocimiento sobre los aspectos que son preguntados, la escasa (o nula) comprensión de las preguntas del cuestionario, fallos de memoria por preguntar aspectos demasiado lejanos en el tiempo, presencia de aquiescencia en las preguntas, etc. (Groves, 1989: 407-445).

3) Los errores producidos por el cuestionario se solucionan con una elaboración cuidadosa del mismo, cumpliendo una serie de requerimientos para la elaboración del cuestionario con el fin de realizar «buenas preguntas», definidas por Fowler como «aquellas que producen respuestas que proporcionan una información fiable y válida en el fenómeno que el investigador quiere describir» (1995: 2).

4) El procedimiento de recogida de datos presenta en ocasiones una gran influencia en las respuestas de los entrevistados, aspecto que no analizaremos aquí puesto que supondría alejarnos del tema principal de este trabajo y por haber sido realizado en otra publicación del autor (Díaz de Rada, 2000). Además, estamos de acuerdo con González Gómez y Padilla García (1999: 92) cuando lo consideran como "fuente indirecta de error».

Estos cuatro aspectos componen el error de medida, sin duda uno de los más importantes y complejos dentro de la investigación social por la gran cantidad de factores que intervienen en su definición. A estos factores añadimos un quinto originado por la situación de la entrevista, y que afecta a todos los anteriores: afecta al entrevistador por la dificultad de realizar la recogida de datos; afecta al entrevistado por la pérdida en la calidad de la información originada cuando los entrevistados son persuadidos a colaborar, se sienten amenazados, etc. (Latiesa, 1994: 335-339); afecta al cuestionario y al procedimiento de recogida de datos cuando éste es encuesta personal o telefónica. 
C) Errores de procesamiento de la información. Cuando finaliza el proceso de recogida de información (trabajo de campo) se revisa la información conseguida, procediendo a repasar la entrevista y cumplimentación correcta del cuestionario, realizar la codificación de los datos recogidos, su grabación y la preparación de éstos para el análisis; situaciones en las cuales pueden volver a cometerse errores.

Los errores de no observación tienen su origen en la falta de cobertura y en la no respuesta de algunos de los elementos de la muestra. El primero se produce cuando no son incluidos determinados elementos de la población objeto de estudio en el proceso de selección muestral (Kish, 1965: 528). La falta de cobertura impide la cooperación de un número de unidades muestrales puesto que determinados individuos no pueden ser seleccionados en la muestra, dificultando con ello la capacidad de inferencia de los hallazgos de la investigación. Este error produce una subestimación en los resultados, cuya amplitud depende de las características de las unidades omitidas (Lavrakas, 1996: 3233). Se desarrollará con profundidad en el siguiente apartado.

El último de los errores señalados es el error producido por la ausencia de respuesta, definida como el «fallo para obtener respuesta en las unidades de análisis (de una población o muestra) por varias razones como la ausencia de una persona en el hogar, rechazo, hogares vacíos, no devolución del cuestionario, omisión de una o más anotaciones en un cuestionario, etc.» (U. S. Bureau of the Census, 1975: 50). Esta definición incluye dos tipos de no respuesta, la producida porque una persona no ha contestado ninguna pregunta del cuestionario, y la generada porque el entrevistado que estaba contestando el cuestionario ha decidido no responder a determinadas cuestiones. La primera de ellas recibe el nombre de «no respuesta total», mientras que la segunda es definida como «no respuesta parcial».

De las numerosas definiciones que existen sobre la «no respuesta total» recogemos la propuesta por Kish (1965: 532) cuando la considera como «el fallo al obtener observaciones (respuestas, medidas) en algunos elementos seleccionados en la muestra", y expone varios factores que contribuyen a aumentar la «no respuesta total»: no existe contacto con el entrevistado, rechazo del mismo, problemas de comunicación entre el entrevistador y el entrevistado, incapacidad para cooperar (enfermedad, problemas de lenguaje, etc.), dificultades de horarios, etc.

\section{EL ERROR DE COBERTURA}

En el apartado anterior se ha señalado que el error de cobertura se produce porque determinadas unidades de la población objeto de estudio no son consideradas dentro de la población de la que se extraerá la muestra. Estas divergencias respecto a la población objeto de estudio precisan el repaso de algunos 
conceptos básicos en teoría muestral. En la definición de la población relevante para la investigación, Groves (1989: 82) distingue cuatro tipos de poblaciones:

- Población de inferencia: formada por el conjunto de personas a estudiar en un momento temporal concreto. Por ejemplo, la población española mayor de 18 años, las personas que tienen derecho a votar, etc.

- Población objetivo: un número determinado de personas que van a ser estudiadas en un momento dado. En algunas ocasiones esta población es similar a la anterior, aunque generalmente suele ser menor que la población de inferencia.

La diferencia entre ambas es realizada por el investigador, que debe explicar las razones por las que no considera determinados colectivos (Groves, 1989: 82). Generalmente, la población objetivo está referida a las personas que viven en hogares principales dentro del área estudiada en un determinado período de tiempo. Es decir, es la población de inferencia menos las personas en instituciones, en bases militares, en lugares remotos del país y aquellas sin domicilio (Groves, 1989: 99).

- Marco poblacional: documento que recoge las personas objeto del estudio. En algunas ocasiones el marco poblacional es una lista de los individuos que viven en un determinado entorno, pero puede ser también un listado de hogares (direcciones, números de teléfono, etc.) e incluso una lista de conglomerados o áreas (secciones censales, bloques de casas, etc.) a los que las personas aparecen unidas por su lugar de residencia (Groves, 1989: 82).

En numerosas ocasiones es muy difícil contar con un documento que refleje con exactitud la población objeto de estudio: en los estudios referidos a colectivos específicos (clientes de un local, visitantes de una zona comercial, etc.) no existe ningún documento que informe sobre las características de la población. Cuando estos colectivos están más acotados, por ejemplo un estudio a los abogados de una comunidad autónoma, es necesario consultar diversas fuentes que proporcionen información sobre ellos (colegios profesionales, asociaciones de abogados, datos sobre las últimas promociones de abogados salidos de la universidad, etc.), llegando incluso a realizar investigaciones para elaborar el marco muestral. A este respecto, Rodríguez Osuna pone el ejemplo de un estudio sobre guarderías realizado por el CIS en noviembre de 1983: "ante la carencia de un censo de guarderías se procedió, por muestreo, a construir el universo» (1991: 18).

Respecto a las investigaciones sobre la población general, o a partes de la misma, se suelen adoptar como marco muestral los censos o padrones generales de población, documentos que en algunas ocasiones presentan grandes deficiencias. De Miguel (1993: 37-40) considera que el Censo de 1991 infraestima la población en medio millón de personas; mientras que Carabaña y otros han detectado que el 17 por 100 de las personas incluidas en el Censo resultaron ilocalizables y un 23 por 100 no pudieron ser contactadas por 
ausencias, ocultamientos, etc. (Carabaña et al., 1992: 91-92). Otros problemas derivados de la utilización de datos censales los expone Manzano (1998: 88).

- Población medida (entrevistada): conjunto de personas que responden a las preguntas formuladas al ser seleccionadas en la muestra.

Del análisis de estas definiciones sobre los tipos de población se desprende que la falta de cobertura puede producirse por una inadecuación entre la población de inferencia y la población objetivo, situación infrecuente, o bien por la existencia de diferencias entre la población objetivo y el marco poblacional. El análisis de esta segunda situación precisará profundizar en los tipos de población incluidos en la población objetivo. Azorín y Sánchez-Crespo (1992: 17-18) definen la población objetivo como aquella que se intenta investigar, la población requerida en términos ideales; aunque diversos motivos (omisiones, duplicaciones, elementos extraños, problemas con los instrumentos de medición, etc.) impiden obtener información de todos los elementos de esta población. Estos autores distinguen dentro de la población objetivo entre la población investigada, la que va a ser realmente estudiada, y la población excluida, que es la diferencia entre ambas, población incluida en la población objetivo pero no en la investigada. Dicho de otro modo, la población investigada es la que queda recogida dentro del marco poblacional, mientras que la población excluida queda fuera del mismo.

Entre las razones de esta diferencia, Lessler y Kalsbeek (1992: 47-52) distinguen una serie de problemas originados al realizar el marco poblacional y otros producidos por una utilización incorrecta de éste. Entre los primeros es posible diferenciar:

1) Ausencias de elementos dentro del marco poblacional. Para solucionar este problema, Sánchez-Crespo aconseja utilizar varios marcos poblacionales, siempre que el número de omisiones sea tal que «merezca la pena incurrir en el costo adicional de preparar un marco complementario al ya existente» (1973: 39) ${ }^{1}$. Además de la utilización de esta estrategia, Lessler y Kalsbeek (1992: 8288 ) insisten también en reducir la población objeto de estudio únicamente a la población incluida en el marco poblacional.

2) Inclusión en el marco poblacional de elementos ajenos a los objetivos del estudio, conocido como sobrecobertura.

3) Personas incluidas varias veces. Hay que tener en cuenta que si una persona está incluida más de una vez aumenta su probabilidad de ser elegida para formar parte de la muestra. Este problema es muy frecuente, por ejemplo, cuando se utilizan guías telefónicas como marco poblacional (Díaz de Rada, 2000). Czaja y Blair (1996: 179) consideran que este problema varía con la edad del marco muestral y la movilidad de la población objeto de estudio.

${ }^{1}$ En cualquier caso, este gasto extraordinario puede ser repartido entre varias investigaciones. 
4) Información incorrecta: marcos poblacionales antiguos, no actualizados, etc.

5) Información auxiliar incorrecta: algunos marcos poblacionales disponen de información auxiliar que puede ser utilizada para muestreos «especiales» o en determinadas técnicas de estimación. Nos referimos concretamente a la información utilizada para la estratificación, para la afijación de la muestra, características de la población relacionadas con el objeto de estudio, etc. El problema es que en ocasiones esta información es incorrecta.

Una utilización inadecuada del marco poblacional puede también ser la causa de las diferencias entre la población objetivo y la investigada. En ocasiones, el investigador puede producir problemas de representatividad porque conscientemente elimina determinados sujetos de su población objetivo, produciendo una falta de cobertura «intencional». Un ejemplo de no cobertura intencional en un sondeo electoral es la eliminación de los residentes en municipios menores de 500 habitantes en la definición del marco muestral, a fin de economizar costos.

Otra situación se produce cuando se realiza un mal uso de los marcos poblacionales que proporcionan información no muy desagregada, es decir, cuando varios elementos de la población tienen únicamente una entrada en el marco muestral (Czaja y Blair, 1996: 179). Será explicado con un ejemplo: supongamos un marco poblacional compuesto no por individuos, sino por los hogares de una comunidad autónoma, aunque incluye también el número de personas que viven en cada hogar. De estas unidades se elige una persona dentro de algunos hogares. Si no se considera el tamaño total de la vivienda se está cometiendo un error de selección puesto que las personas que viven en hogares unipersonales tendrán más probabilidad de pertenecer a la muestra. Es decir, se considera que la probabilidad que tiene una persona de ser elegida en la muestra es la misma que la probabilidad de elección de cada hogar. Para solucionar este problema, Czaja y Blair proponen, una vez seleccionada una persona de un hogar, aplicarle en el proceso de análisis un coeficiente de ponderación en función del número de personas que residen en ese hogar (1996: 179).

Todo lo expuesto hasta el momento carecería de interés si no existieran diferencias en las características de un fenómeno al no considerar la población «no cubierta». Un primer objetivo por el que se realiza una investigación mediante encuesta es obtener un conocimiento de una población, y por ello seleccionamos una parte que sea representativa de ésta. El objetivo final es realizar inferencias a esa población, tratando de conocer el número de escaños que obtendrá un partido político, el número de hogares que han visto un determinado programa de televisión, etc. El gran problema que produce la no cobertura es la pérdida de representación que se comete al obviar un determinado tipo de personas, de modo que este descenso en la representatividad se traduce en una pérdida de capacidad de inferencia de la muestra, produciendo un sesgo en el proceso de inferencia. 
El error de cobertura se refiere así a la diferencia entre los valores calculados en el marco poblacional y estos valores en la población objetivo, diferencia que depende del número de unidades omitidas y de las características de éstas.

\section{FIGURA 1}

\section{Error de cobertura}

$$
Y=\frac{N_{c}}{N} Y_{c}+\frac{N_{n c}}{N} Y_{n c}
$$

Donde:

$Y=$ valor del estadístico en la población objetivo

$N_{c}=$ número de elementos de la población objetivo incluidos en el marco poblacional

$N_{n c}^{c}=$ número de elementos de la población objetivo excluidos en el marco poblacional

$N=$ tamaño de la población objetivo

$Y_{c}=$ valor del estadístico en las personas incluidas en el marco poblacional

$Y_{n c}^{c}=$ valor del estadístico en aquellos no incluidos en el marco poblacional

FUENTE: Groves (1989: 54-85).

La expresión anterior está indicando, en definitiva, que la cuantía del error de cobertura depende de dos factores: la magnitud de la cobertura $\left(N_{n c} / N\right)$ y las diferencias entre la población cubierta y no cubierta (diferencias entre $Y_{c}$ e $Y_{n c}$ ). En el ámbito que nos ocupa, las encuestas telefónicas, esto quiere decir que aunque un elevado número de personas carezcan de teléfono, el error de cobertura será pequeño si estas personas no presentan muchas diferencias respecto a las personas con teléfono. Del mismo modo, si las personas que tienen teléfono son muy distintas a las personas con teléfono, un escaso número de personas sin teléfono puede producir sesgos importantes en el momento de realizar inferencias a la población objeto de estudio.

Un gran número de investigaciones han demostrado que las personas que viven en hogares sin teléfono presentan grandes diferencias en variables de interés en el análisis sociológico, como tendremos ocasión de demostrar en el apartado siguiente.

Sin embargo, el problema de la cobertura telefónica no se reduce únicamente a las personas sin teléfono, sino que es preciso considerar también aquellos colectivos que presentan problemas de accesibilidad por la dificultad de «encontrar»su número de teléfono, siempre que el marco poblacional sea una guía de teléfonos y no un sistema de números aleatorios. Thomas y Purdon (1994: 1) destacan que en el Reino Unido una de cada cuatro personas sin teléfono tiene números no listados en las guías. Aunque la población sin teléfono está disminuyendo, desde su punto de vista, los hogares con teléfonos «no 
listados» están incrementándose: los «robinsones», que denomina José Ignacio Wert (1994: 181). Basados en datos de Coditel y Telefónica, Bosch y Torrente (1993: 92) estiman el número de robinsones en nuestro país en 100.000 personas, aunque consideran que no afectan sustancialmente a la extrapolación de resultados por tener una característica común: la mayoría son personalidades públicas. Otro grupo que presenta problemas de localización son las personas que aparecen en las guías pero que ya no tienen ese teléfono porque se han dado de baja o porque ya no viven ahí. Thomas y Purdon señalan que en el Reino Unido estas personas son fundamentalmente jóvenes, viven en ciudades y presentan gran movilidad espacial (Thomas y Purdon, 1994: 2); mientras que, en Estados Unidos, Keeter considera que estos colectivos presentan características similares a las personas sin teléfono (1995: 213-215). La escasa movilidad residencial en la sociedad española nos lleva a considerar que este problema no es muy importante en nuestro país.

Tras esta exposición del error de cobertura en las investigaciones mediante encuestas pasaremos a definir específicamente los problemas de cobertura planteados por las encuestas telefónicas en distintos países.

\section{EL ERROR DE COBERTURA DE LAS ENCUESTAS TELEFÓNICAS EN DISTINTAS SOCIEDADES}

En un trabajo sobre el análisis de no cobertura en las encuestas telefónicas, Trewin y Lee (1988: 9-24) dan cuenta de los problemas para conseguir información fiable y comparable sobre la tasa de posesión de teléfonos en distintas sociedades. Así, mientras que algunos países recogen información sobre el número de hogares con teléfono, otros presentan estadísticas sobre número de teléfonos por 1.000 habitantes. En cualquier caso, este trabajo ha llegado a ser considerado como un clásico en el análisis de la cobertura, de modo que lo utilizaremos aquí para analizar el error de cobertura a nivel mundial. De acuerdo que los datos que presentan pueden estar ya muy desfasados, pero también es verdad que el objetivo del presente artículo no es analizar la cobertura a nivel mundial, sino conocer los problemas de cobertura de las encuestas telefónicas que se realizan en nuestro país.

De este modo, el trabajo de Trewin y Lee nos proporcionará una serie de "pistas» sobre las características de los hogares sin teléfono, a fin de analizar - en un segundo momento - hasta qué punto estos rasgos se producen también en la sociedad española. Trewin y Lee (1988: 14-21) consideran nueve características a la hora de definir la población sin teléfono en diversos países $^{2}$ :

2 Los países analizados fueron: Alemania Federal, Australia, Austria, Canadá, Dinamarca, España, Estados Unidos, Finlandia, Francia, Grecia, Hong Kong, Hungría, Irlanda, Israel, Italia, México, Noruega, Nueva Zelanda, Países Bajos, Polonia, Reino Unido y Suecia. 
- Características geográficas: la cobertura varía según las regiones, siendo menor en las regiones más lejanas. Trewin y Lee (1988: 14) no explican si se refiere a lejanía del centro del país respecto a vías importantes de comunicación, respecto a la capital del país, etc.

- Tamaño del municipio: la cobertura telefónica es mayor en las ciudades que en el mundo rural, a excepción de algunos países escandinavos.

- Tamaño del hogar: la cobertura es inferior en los hogares unifamiliares, y crece a medida que aumenta el tamaño de la familia. Esta pauta es similar en todos los países analizados.

- Ingresos: los hogares sin teléfono tienen un nivel de ingresos inferior a los hogares con teléfono. En la página 19 presentan un gráfico donde se representan las desviaciones típicas de los ingresos en relación con la posesión del teléfono, que les permite señalar que en las sociedades más desarrolladas estas diferencias de cobertura en función de los ingresos son menores.

- Relación con la actividad: la cobertura es inferior en los hogares cuyo cabeza de familia está desempleado y en aquellos hogares con personas desempleadas. Esta tendencia se observa en todos los países analizados.

- Ocupación: la cobertura es alta cuando el cabeza de familia tiene una ocupación especializada.

- Edad del cabeza de familia: el análisis de la mayor parte de los países desvela que la cobertura aumenta con la edad del cabeza de familia, hasta que éste alcanza los 65 años. A partir de este momento la cobertura disminuye, a excepción de Estados Unidos.

- Vivienda: la cobertura es baja para viviendas en régimen de alquiler. La cobertura disminuye con la edad de la vivienda. radas.

- Estado civil: la no cobertura es mayor en las personas solteras o sepa-

\section{A) Caracteristicas del error de no cobertura en los Estados Unidos}

Tras este análisis de la cobertura a nivel mundial centraremos nuestra atención al país donde nacen las encuestas telefónicas y cuya utilización está más extendida. Como se ha señalado en las primeras líneas de este trabajo, más del 70 por 100 de las investigaciones que se realizan en los Estados Unidos utilizan la encuesta telefónica.

El análisis de la cobertura en los Estados Unidos, el país donde más se han desarrollado este tipo de encuestas, pudiera ser utilizado para ver «hasta qué niveles» puede admitirse la no cobertura. Así, la evolución histórica de la cobertura telefónica en este país, unido a la progresiva utilización de las encuestas telefónicas, será utilizada para comparar la situación española actual con la situación americana de hace «X» años. Si en el momento actual la cobertura del teléfono en España es similar a la cobertura americana hace veinte años, por ejemplo, considerar el número de entrevistas telefónicas realizadas en Estados Unidos hace 
veinte años podría utilizarse como un indicador de la adecuación del número de entrevistas telefónicas realizadas en España en la actualidad.

De modo que comenzaremos analizando la primera parte de la expresión mostrada en la figura 1, que se preocupa de cuantificar el número de personas no incluidas en la muestra. El análisis de la tabla 2 desvela que en 1963 un 80,3 por 100 de la población americana disponía de teléfono en su hogar, porcentaje que se amplía considerablemente en los siguientes veintidós años. En 1975 tan sólo el 9,6 por 100 de la población norteamericana carece de este equipamiento, experimentando una ligera reducción en los siguientes diez años como consecuencia del elevado nivel de desempleo tras la crisis económica de mediados de la década de los setenta (Thornberry y Massey, 1988: 27). Así, en 1986 el 94 por 100 de la población dispone de teléfono en su hogar.

TABLA 2

Porcentaje de hogares sin teléfono en los Estados Unidos (Ratio hogares sin teléfono/total de hogares)

\begin{tabular}{|c|c|}
\hline Аño 1963 & 19,7 \\
\hline Año 1970 & 11,7 \\
\hline Año 1975 & 9,6 \\
\hline Аño 1980 & 7,5 \\
\hline Año 1985 & 7,2 \\
\hline Año 1986 & 6,0 \\
\hline
\end{tabular}

FueNTE: Thornberry y Massey (1988: 28).

Tras conocer la magnitud de la población cubierta se procederá a analizar las diferencias entre la población cubierta y no cubierta; es decir, el segundo elemento de la expresión mostrada en la figura 1. El análisis de la tabla 3 muestra que la cobertura telefónica es menor en los hogares del sur en los tres períodos considerados, experimentando un ligero crecimiento del 4 por 100 entre 1975 y 1985 . En relación al hábitat la cobertura telefónica se ha incrementado notablemente en los diez años analizados, principalmente en la población rural no agrícola. Thornberry y Massey (1988: 28) explican este fenómeno por el descenso en la población rural dedicada al sector primario y el desarrollo de la tecnología telefónica.

El análisis de las características sociodemográficas de los entrevistados desvela que la cobertura telefónica es menor en los colectivos menores de 24 años, mientras que las personas de más edad son las más cubiertas telefónicamente. Una menor posesión de teléfono se aprecia también en los hogares con personas de raza "no blanca", en los hogares donde los entrevistados están desempleados y separados. En relación a estos últimos, Thornberry y Massey (1988: 
30) señalan que el origen puede estar en su reciente cambio de estilo de vida y nivel económico. Respecto al nivel educativo del cabeza de familia y los ingresos del hogar, a medida que aumentan disminuye el número de hogares sin teléfono.

\section{TABLA 3}

Características de los hogares sin teléfono en los Estados Unidos (Porcentajes de hogares sin teléfono)

\begin{tabular}{|c|c|c|c|}
\hline & 1975 & 1980 & $1985-86$ \\
\hline \multicolumn{4}{|l|}{ Región: } \\
\hline 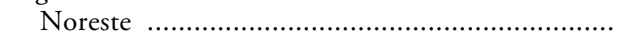 & 7,1 & 5,2 & 4,5 \\
\hline Medio Oeste y Norte Central ................................... & 6,6 & 5,3 & 5,3 \\
\hline Sur & 14,0 & 11,5 & 10,4 \\
\hline Oeste & 9,7 & 6,2 & 6,9 \\
\hline \multicolumn{4}{|l|}{ Hábitat: } \\
\hline Urbano & 8,3 & 7,0 & 6,4 \\
\hline Rural agrícola & 7,9 & 4,5 & 4,5 \\
\hline 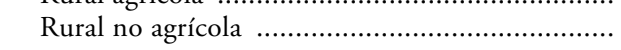 & 13,9 & 9,1 & 9,9 \\
\hline \multicolumn{4}{|l|}{ Número de personas en el hogar: } \\
\hline 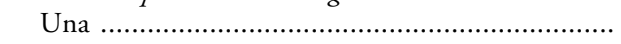 & 15,4 & 11,7 & 9,9 \\
\hline Dos & 7,8 & 5,5 & 5,2 \\
\hline 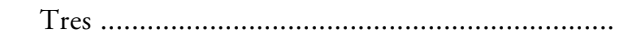 & 5,8 & 6,5 & 6,4 \\
\hline Cuatro & 6,7 & 5,8 & 6,1 \\
\hline 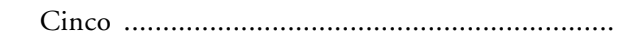 & 7,7 & 6,9 & 7,9 \\
\hline Seis & 10,5 & 8,7 & 10,8 \\
\hline 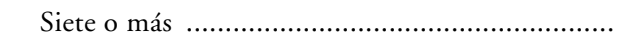 & 14,6 & 12,0 & 16,3 \\
\hline \multicolumn{4}{|l|}{ Sexo: } \\
\hline 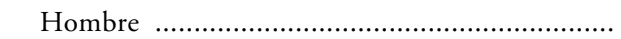 & 9,8 & 7,6 & 7,6 \\
\hline Mujer …… & 8,6 & 6,6 & 6,7 \\
\hline \multicolumn{4}{|l|}{ Edad: } \\
\hline 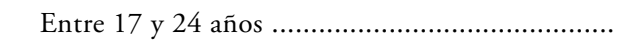 & 13,8 & 11,3 & 11,2 \\
\hline 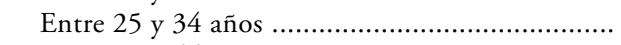 & 9,6 & 7,7 & 8,0 \\
\hline 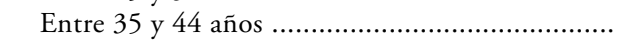 & 7,1 & 5,0 & 5,0 \\
\hline 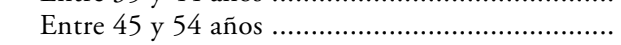 & 5,5 & 4,2 & 4,2 \\
\hline Entre 55 y 64 años & 5,3 & 3,6 & 3,7 \\
\hline 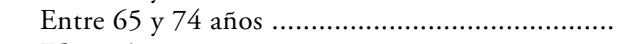 & 5,6 & 4,0 & 3,2 \\
\hline 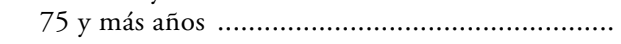 & 6,3 & 4,2 & 3,3 \\
\hline \multicolumn{4}{|l|}{$R a z a:$} \\
\hline 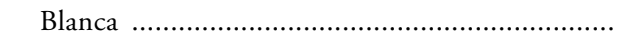 & 7,6 & 5,8 & 5,8 \\
\hline Negra & 20,4 & 16,8 & 15,6 \\
\hline Otra & 11,6 & 9,4 & 10,9 \\
\hline
\end{tabular}




\section{TABLA 3 (continuación)}

\section{Características de los hogares sin teléfono en los Estados Unidos}

(Porcentajes de hogares sin teléfono)

\begin{tabular}{|c|c|c|c|}
\hline & 1975 & 1980 & $1985-86$ \\
\hline \multicolumn{4}{|l|}{ Estado civil: } \\
\hline 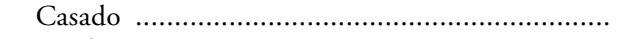 & 7,0 & 4,9 & 5,0 \\
\hline Viudo & 7,6 & 5,1 & 4,5 \\
\hline 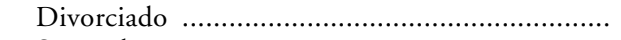 & 12,9 & 11,2 & 9,8 \\
\hline 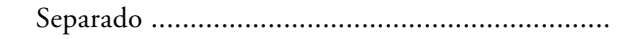 & 25,3 & 18,0 & 18,8 \\
\hline Soltero ….......................... & 10,2 & 8,6 & 8,1 \\
\hline \multicolumn{4}{|l|}{ Situación de empleo: } \\
\hline 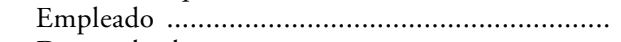 & 6,9 & 5,4 & 5,2 \\
\hline 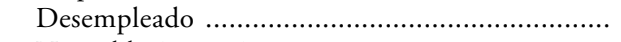 & 16,0 & 13,5 & 16,0 \\
\hline 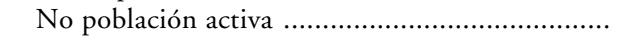 & 9,3 & 7,2 & 7,2 \\
\hline \multicolumn{4}{|l|}{ Nivel educativo del cabeza de familia: } \\
\hline 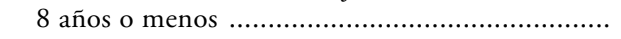 & - & - & 18,1 \\
\hline 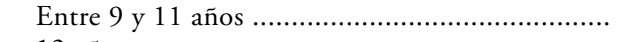 & - & - & 19,2 \\
\hline 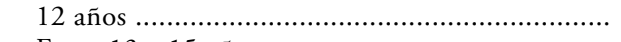 & - & - & 8,0 \\
\hline 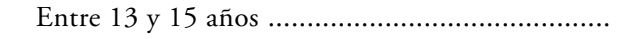 & - & - & 4,1 \\
\hline 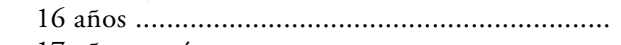 & - & - & 1,8 \\
\hline 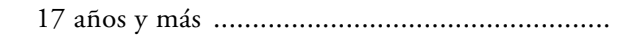 & - & - & 0,8 \\
\hline \multicolumn{4}{|l|}{ Ingresos del hogar: } \\
\hline 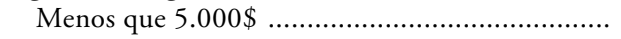 & - & - & 29,2 \\
\hline 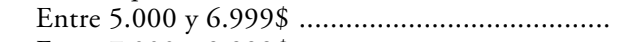 & - & - & 20,0 \\
\hline 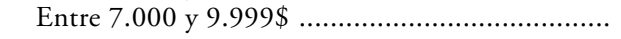 & - & - & 18,1 \\
\hline 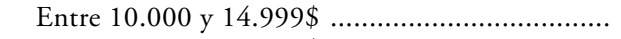 & - & - & 12,9 \\
\hline 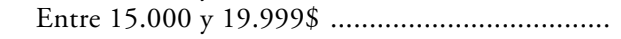 & - & - & 7,7 \\
\hline 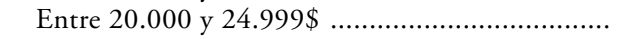 & - & - & 3,6 \\
\hline 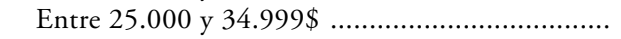 & - & - & 1,8 \\
\hline 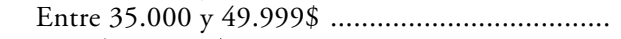 & - & - & 0,9 \\
\hline Más de $50.000 \$$ & - & - & 0,4 \\
\hline 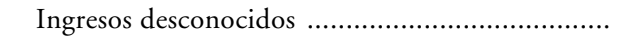 & - & - & 8,0 \\
\hline
\end{tabular}

Fuente: Thornberry y Massey, 1988: 29, 30 y 34.

Basado en datos obtenidos de la encuesta general de población, Smith (1990: 385) señala que el nivel de ingresos familiares y el nivel educativo son las variables que más influyen en la posesión del teléfono, aunque sin despreciar otras variables relacionadas con el estilo de vida y el nivel cultural de los entrevistados. Desde su punto de vista, los individuos sin teléfono se caracterizan por vivir en viviendas no propias, baja frecuencia de asistencia a la iglesia y por pertenecer a grupos pequeños. Groves (1990: 228-229) recoge información de diversas investigaciones que le llevan a afirmar que las personas sin teléfono tienen más enfermedades que el resto, realizan menos visitas al médico, están menos cubiertas por seguros médicos, son víctimas de más crímenes y 
presentan tasas de desempleo superiores al resto de la población. En el Reino Unido, Thomas y Purdon (1994) señalan que la mayor parte de este colectivo está formado por personas de muy baja condición social.

\section{B) La situación en España}

Anteriormente se ha señalado que un 29 por 100 de las encuestas realizadas en nuestro país se realizaron mediante entrevistas telefónicas. El análisis de la investigación Industria de los Estudios de Mercado en España permite actualizar estos resultados al desvelar que un 44 por 100 de los casi ocho millones (7.855.000) de encuestas realizadas en 1998 se llevaron a cabo mediante el teléfono, que supone un notable incremento respecto al número de entrevistas realizadas en los años anteriores: en 1997, el 28 por 100 de las encuestas realizadas (8.878.000) utilizaron el teléfono. En 1996, el 29 por 100 de las 6.706 .000 entrevistas se realizaron mediante este procedimiento (Alós, 1997: 74; 1998: 62; 1999: 75).

Ahora bien, la adecuada utilización de estas entrevistas precisa que la mayor parte de la población esté cubierta telefónicamente, aspecto al que se dedicará este apartado. En la tabla 4 se muestra que desde 1960 la cobertura telefónica en España ha aumentado considerablemente, si bien no es hasta 1981 cuando la mitad de la población española dispone de teléfono. Diez años más tarde, más de las tres cuartas partes de los hogares españoles tienen teléfono, porcentaje que llega al 83 por 100 en 1998.

\section{TABLA 4}

Porcentaje de hogares con teléfono en España

(Ratio hogares con teléfono/total de hogares)

\begin{tabular}{|c|c|c|}
\hline Año 1960 & & $12,0^{\mathrm{a}}$ \\
\hline Año 1968 & ...... & $19,0^{\mathrm{b}}$ \\
\hline Año 1975 & & $33,8^{c}$ \\
\hline Año 1981 & & $51,0^{d}$ \\
\hline Año 1991 & . & $76,9^{\mathrm{e}}$ \\
\hline Año 1998 & & $82,9^{f}$ \\
\hline
\end{tabular}

FUENTES:

a Selecciones del Reader's Digest, Retrato de dos Familias (1960).

b INE, Encuesta de equipamiento y nivel cultural de las Familias, Madrid: INE, 1968.

c INE, Encuesta de equipamiento y nivel cultural de las familias (abril 1975), vol. I, Madrid, 1976.

' INE, Censo de viviendas 1981, tomo 1: Resultados nacionales, Madrid, INE, 1987.

- INE, Encuestas de Presupuestos Familiares 1990/91.

f CIS, Los comportamientos económicos de los españoles: ahorro y consumo. 
Además del porcentaje de población cubierta telefónicamente, tan importante o más es conocer las diferencias entre la población cubierta y no cubierta, con el fin de saber si puede considerarse que la población sin teléfono es similar a la que dispone de este equipamiento (figura 1). La tabla 5 muestra grandes diferencias entre ambos colectivos prácticamente en todas las variables consideradas. La posesión de teléfono en 1998 es superior al promedio nacional en las ciudades intermedias (entre 100.001 y 400.000 habitantes) y en las ciudades de más de un millón de habitantes, así como en las Comunidades de Navarra, Asturias, País Vasco, Madrid, Cataluña, Castilla y León y Comunidad Valenciana ${ }^{3}$. En cuanto a los rasgos sociodemográficos, los entrevistados con mayor tasa de teléfono viven en hogares de 4 y más miembros, edades entre 45 y 55 años y mayores de 75 años, casados, nivel educativo de escuela universitaria o universidad, ideología de izquierda y centro, e ingresos mensuales superiores a las 200.000 pesetas.

En relación a la evolución respecto a 1991 es preciso destacar el importante incremento en la posesión del teléfono en los municipios menores de 10.000 habitantes y en los que tienen entre 50.001 y 100.000 habitantes, así como en las Comunidades de Baleares, las dos Castillas, Extremadura y Murcia. Los hogares con más miembros han aumentado sustancialmente la posesión del teléfono en los últimos años, así como los entrevistados entre 25 y 54 años y los mayores de 75 años. Esto mismo sucede en las personas sin estudios y con estudios de FP segundo grado, ideología de izquierda y centro izquierda, $\mathrm{y}$ con ingresos inferiores a las 100.000 pesetas.

Por contra, la tasa de posesión del teléfono es menor en los asentamientos de población entre los 2.001 y 50.000 habitantes, así como en las Comunidades de Andalucía, Canarias, Extremadura y Galicia. Los entrevistados sin teléfono destacan por vivir en hogares de uno o dos miembros, edad entre 25 y 34 años, solteros y separados, estudios primarios, no responden la pregunta sobre la ideología, ingresos inferiores a las 150.000 pesetas y parados.

\section{TABLA 5}

Caracteristicas de los hogares con teléfono en España (\%)

\begin{tabular}{|c|c|c|}
\hline & 1991 & 1998 \\
\hline \multicolumn{3}{|l|}{ Comunidad Autónoma ${ }^{* *_{a}}$ : } \\
\hline 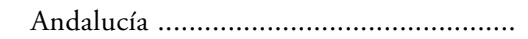 & 64,2 & 66,4 \\
\hline 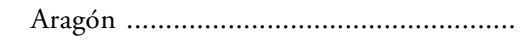 & 82,3 & 84,6 \\
\hline 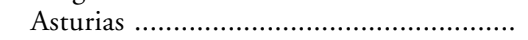 & 94,0 & 93,2 \\
\hline 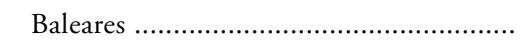 & 68,0 & 87,2 \\
\hline
\end{tabular}




\section{TABLA 5 (continuación)}

\section{Caracteristicas de los hogares con teléfono en España (\%)}

\begin{tabular}{|c|c|c|}
\hline & 1991 & 1998 \\
\hline Cataluña ...... & 90,4 & 89,6 \\
\hline Canarias & 67,7 & 68,7 \\
\hline 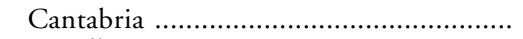 & 77,0 & 79,4 \\
\hline 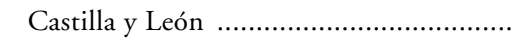 & 75,4 & 88,4 \\
\hline 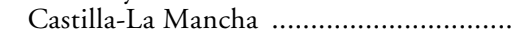 & 65,1 & 85,2 \\
\hline Extremadura & 64,7 & 77,6 \\
\hline Galicia & 75,0 & 78,1 \\
\hline 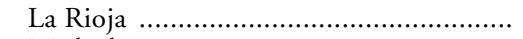 & 87,8 & 88,2 \\
\hline 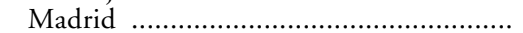 & 92,4 & 90,2 \\
\hline 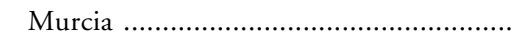 & 62,8 & 81,8 \\
\hline 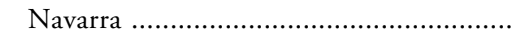 & 93,5 & 94,1 \\
\hline 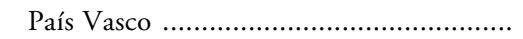 & 91,2 & 90,6 \\
\hline Comunidad Valenciana ......................... & 68,8 & 87,8 \\
\hline \multicolumn{3}{|l|}{ Hábitat**: } \\
\hline Menos de 2.000 habitantes ........................ & 67,7 & 80,0 \\
\hline De 2.001 a 10.000 habitantes ................. & 67,9 & 78,2 \\
\hline De 10.001 a 50.000 habitantes ................ & 74,9 & 79,8 \\
\hline De 50.001 a 100.000 habitantes .............. & 74,6 & 81,3 \\
\hline De 100.001 a 250.000 habitantes ........... & 86,9 & $87,1^{\mathrm{b}}$ \\
\hline Más de 250.000 habitantes ..................... & 77,1 & $81,2^{\mathrm{c}}$ \\
\hline Madrid y Barcelona .............................. & 97,5 & $91,3^{\mathrm{d}}$ \\
\hline \multicolumn{3}{|l|}{ Número de miembros en el hogar*: } \\
\hline Uno .................... & 68,1 & 67,6 \\
\hline Dos & 75,8 & 79,7 \\
\hline 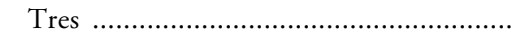 & 74,6 & 84,0 \\
\hline 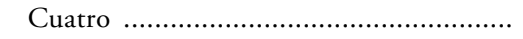 & 81,9 & 86,4 \\
\hline Cinco & 82,1 & 86,9 \\
\hline Seis & 86,7 & 85,4 \\
\hline 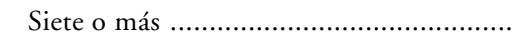 & 71,2 & 81,5 \\
\hline \multicolumn{3}{|l|}{ Sexo entrevistado: } \\
\hline 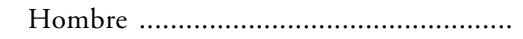 & 75,1 & 81,6 \\
\hline 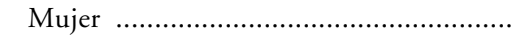 & 80,6 & 84,2 \\
\hline \multicolumn{3}{|l|}{ Edad*: } \\
\hline De 18 a 24 años & 80,2 & 82,7 \\
\hline 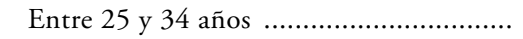 & 71,4 & 79,2 \\
\hline Entre 35 y 44 años & 76,1 & 82,6 \\
\hline 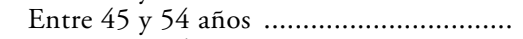 & 78,1 & 86,5 \\
\hline 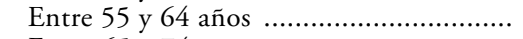 & 81,8 & 81,8 \\
\hline 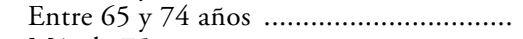 & 85,9 & 85,1 \\
\hline 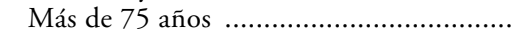 & 72,7 & 86,7 \\
\hline \multicolumn{3}{|l|}{ Estado civil**: } \\
\hline 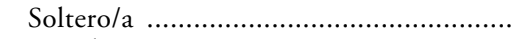 & 79,4 & 80,4 \\
\hline 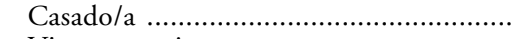 & 77,4 & 84,8 \\
\hline Vive en pareja & 69,9 & - \\
\hline 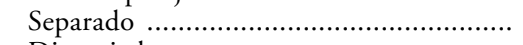 & 73,7 & 61,0 \\
\hline Divorciado & 89,4 & 78,3 \\
\hline Viudo/a & 78,5 & 82,7 \\
\hline
\end{tabular}




\section{TABLA 5 (continuación)}

Características de los hogares con teléfono en España (\%)

\begin{tabular}{|c|c|c|}
\hline & 1991 & 1998 \\
\hline \multicolumn{3}{|l|}{ Nivel educativo entrevistado*: } \\
\hline Sin estudios & 68,6 & 82,4 \\
\hline 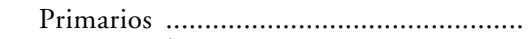 & 77,1 & 80,2 \\
\hline 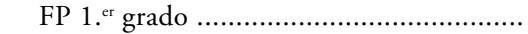 & 84,8 & 84,0 \\
\hline FP 2.0 grado & 77,2 & 86,6 \\
\hline 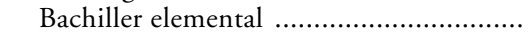 & 80,6 & 82,3 \\
\hline Bachiller superior & 90,2 & 85,6 \\
\hline Grado medio (escuela universitaria) ........ & 86,2 & 89,6 \\
\hline 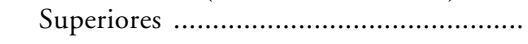 & 93,8 & 90,4 \\
\hline \multicolumn{3}{|l|}{ Ideologia ${ }^{* *}$ : } \\
\hline 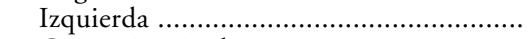 & 76,3 & 87,1 \\
\hline Centro-izquierda & 77,0 & 80,9 \\
\hline Centro & 83,7 & 86,4 \\
\hline Centro-derecha & 81,0 & 84,2 \\
\hline 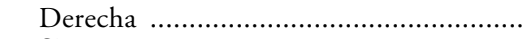 & 88,7 & 86,1 \\
\hline 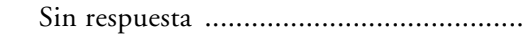 & 75,2 & 78,7 \\
\hline \multicolumn{3}{|l|}{ Ingresos mensuales del hogar**: } \\
\hline 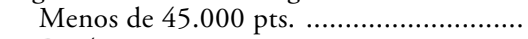 & 61,8 & $58,8^{\circ}$ \\
\hline 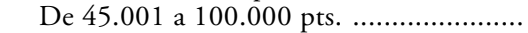 & 71,2 & 77,2 \\
\hline De 100.001 a 150.000 pts. .......................... & 79,4 & 79,7 \\
\hline De 150.001 a 200.000 pts. ..................... & 89,9 & 83,2 \\
\hline De 200.001 a 275.000 pts. ...................... & 98,0 & $92,2^{\mathrm{f}}$ \\
\hline 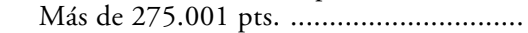 & 96,1 & 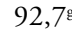 \\
\hline Ingresos desconocidos …............................ & 79,5 & 84,1 \\
\hline \multicolumn{3}{|l|}{ Situación de empleo: } \\
\hline Población activa & 77,0 & 81,7 \\
\hline Población no activa .................................... & 81,4 & 84,3 \\
\hline \multicolumn{3}{|l|}{ Relación con la actividad: } \\
\hline 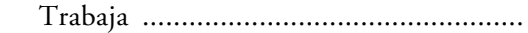 & 76,1 & 83,3 \\
\hline 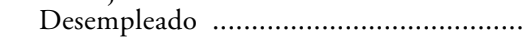 & 77,0 & 75,7 \\
\hline
\end{tabular}

NOTAS:

* V de Cramer significativa al 0,05. $\quad * *$ V de Cramer significativa al 0,01.

a Aconsejamos prudencia en la interpretación de los resultados de esta variable para las comunidades uniprovinciales o escasamente pobladas, por los escasos tamaños muestrales.

b De 100.001 a 400.000 habitantes.

c De 400.001 a 1.000 .000 de habitantes.

d Más de 1.000.000 de habitantes.

e Hasta 50.000 pesetas.

De 200.001 a 300.000 pesetas.

g Más de 300.001 pesetas.

FUENTES: Fundación CIRES (1991) ; Centro de Investigaciones Sociológicas (1998)

${ }^{4}$ Ficha técnica: Estudio sobre estilos de vida, realizado en octubre de 1991 mediante 1.200 entrevistas personales en todo el territorio nacional.

Muestra aleatoria estratificada por Comunidades Autónomas y municipios según su tamaño, representativa de la población española de 18 y más años, con un error muestral del $\pm 2,89$ por 100 para $\mathrm{p}=\mathrm{q}$ y un margen de confianza del 95,5 por 100 .

5 Ficha técnica: Estudio sobre los comportamientos económicos de los españoles (consumo y aho- 


\section{VARIABLES DETERMINANTES DEL ERROR DE COBERTURA}

En el apartado anterior se ha mostrado cómo diversas variables influyen en la posesión del teléfono, llegando a plantear una tipología de las personas que poseen y que carecen de este equipamiento. Con este apartado se pretende avanzar en esta problemática localizando qué variables influyen más en la posesión del teléfono, con el objetivo de identificar con precisión los colectivos con mayor y menor cobertura telefónica.

Para conseguir estos propósitos utilizaremos la técnica estadística del Análisis Automático de la Interacción (Automatic Interaction Detector-AID), desarrollado por Sonquist y Morgan en los años sesenta. La variable dependiente, o situación a predecir, es la posesión del teléfono en el hogar, y las variables independientes o predictoras todas las que se han mostrado en la tabla 5. El carácter nominal de la variable dependiente aconseja utilizar una versión del AID desarrollada por Kass en 1980, y que recibe el nombre de CHAID (Chi-Square Automatic Interaction Detector). La principal diferencia con el AID es que éste tiene una variable dependiente de intervalo o razón, mientras que el CHAID utiliza variables dependientes nominales u ordinales ${ }^{6}$.

La técnica que utilizaremos aquí, junto con el AID, THAID, etc., se conoce también como Análisis de Segmentación puesto que su fin es "polarizar» las frecuencias de las categorías de una variable dependiente cualitativa seleccionando los sujetos que cumplan determinadas características: se trata de preguntarnos si es posible identificar segmentos de población dentro de los cuales la variación de la variable dependiente sea significativamente menor que en el conjunto de la muestra. Una de las mayores ventajas de estas técnicas es su enorme facilidad para descubrir el efecto de la interacción, cómo la influencia de una variable independiente varía según el valor de otra variable independiente.

En la figura 2 se muestra la estructura «arbólea» resultado del análisis de segmentación. Tres interpretaciones pueden realizarse de esta figura: la primera consiste en analizar el orden en que las variables son introducidas en el análisis, en la segunda la atención se concentra en las asociaciones entre variables y categorías de variables y, por último, el análisis de los «tipos» resultantes.

Comenzaremos con la primera de ellas, con el fin de conocer qué variables afectan más a la cobertura telefónica. Como puede observarse, el nivel de

rro), realizado en octubre de 1998 mediante 2.500 entrevistas personales en todo el territorio nacional.

Muestreo polietáptico, estratificado por conglomerados, con selección de las unidades primarias de muestreo (municipios) y de las unidades secundarias (secciones) de forma aleatoria proporcional, y de las unidades últimas (individuos) por rutas aleatorias y cuotas de sexo y edad. Para un nivel de confianza del 95,5 por 100 y p=q, el error muestral es de $\pm 1,96$ por 100 para el conjunto de la muestra y en el supuesto de muestreo aleatorio simple.

${ }^{6}$ A los interesados a un análisis en detalle del funcionamiento de esta técnica aconsejamos la lectura de Escobar (1998: 13-50). 


\section{FIGURA 2}

\section{Resultados del análisis de segmentación (CHAID)}

TOTAL: 2.500

Porcentaje entrevistados con teléfono: 82,9

NIVEL DE INGRESOS (en miles de pesetas)

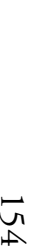

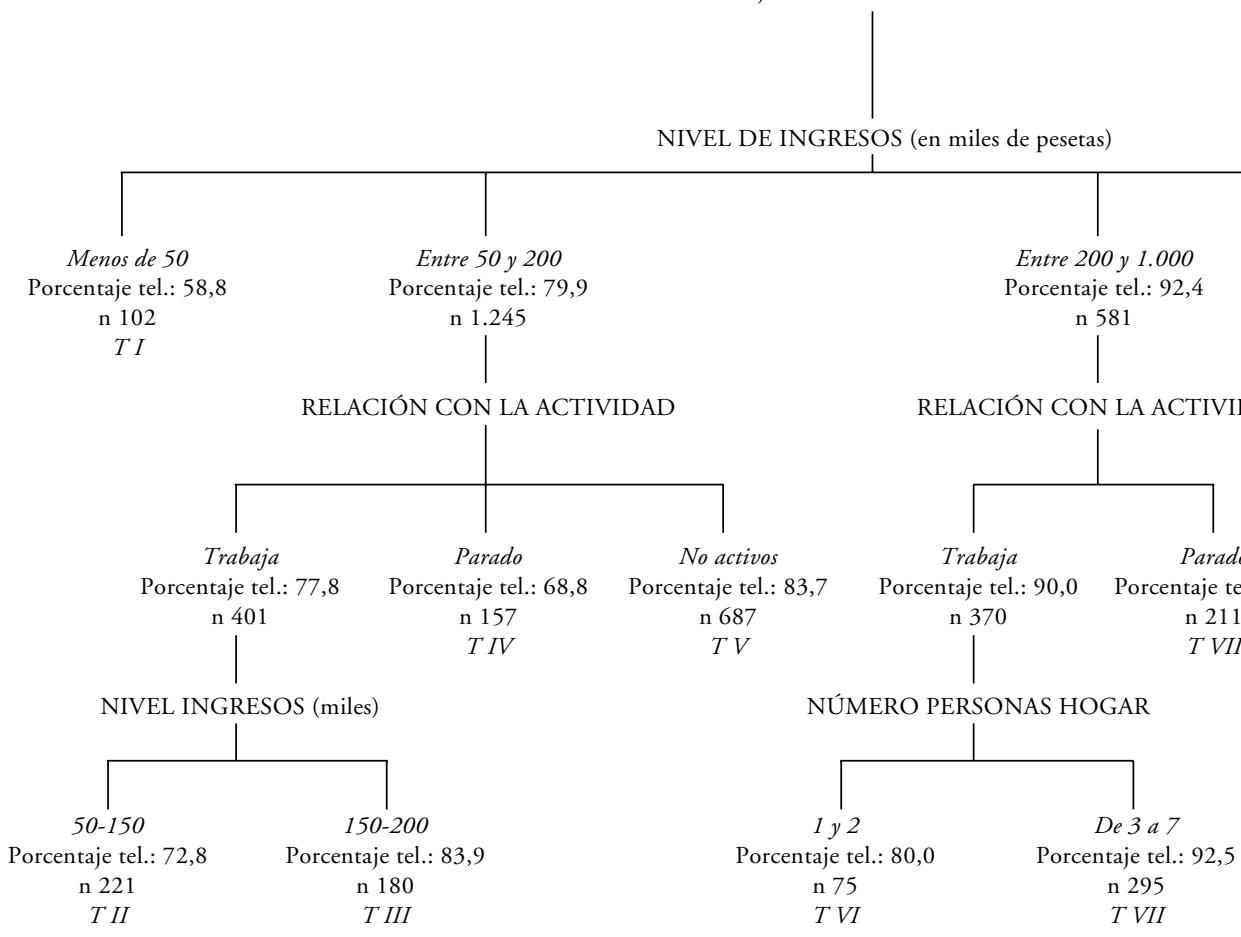


ingresos es la variable que mejor segmenta la posesión de teléfono en el hogar, que aparece dividida en cuatro grupos: menores de 50.000 pesetas al mes, entre 50.001 y 200.000 pesetas, más de 200.001 pesetas mensuales y, por último, los que no saben lo que ganan o no han contestado a esa pregunta. En el subgrupo con menores ingresos el 60 por 100 dispone de teléfono en su hogar, cifra que se eleva al 80 por 100 en el segundo y al 92,4 por 100 en el tercero. En el subgrupo de entrevistados que no responden a la pregunta sobre los ingresos la tasa de posesión del teléfono es del 84 por 100.

El subgrupo de ingresos entre 50.001 y 200.000 pesetas es dividido por la relación con la actividad, hecho que permite conocer que el 69 por 100 de los parados con estos ingresos disponen de teléfono, ratio que aumenta al 78 por 100 entre los que trabajan y al 84 por 100 entre los entrevistados que no forman parte de la población activa. Las personas que trabajan vuelven a ser divididas por el nivel de ingresos del hogar, personas con ingresos inferiores y superiores a las 150.000 pesetas. De los primeros, el 73 por 100 dispone de teléfono en su hogar, porcentaje que aumenta hasta el 84 por 100 en los segundos.

El tercer subgrupo de ingresos, mayores de 200.001 pesetas, vuelve a ser dividido por la relación con la actividad. De las personas que están en paro el 97 por 100 dispone de teléfono, porcentaje que se reduce al 90 por 100 en el caso de las personas que trabajan. Las personas que trabajan presentan diferencias en su cobertura telefónica atendiendo al tamaño de su familia: el 80 por 100 de las familias de uno o dos miembros dispone de teléfono, ratio que aumenta hasta el 92 por 100 entre las familias de tres y más miembros.

Por último, los entrevistados que no han contestado al nivel de ingresos son divididos atendiendo al tamaño del municipio donde residen. Por un lado, los municipios más pequeños y los más grandes, con una tasa de cobertura telefónica del 91,4 por 100 , y los municipios entre 2.001 y 1.000 .000 habitantes, con una tasa del 82 por 100 .

La combinación de cada uno de estos rasgos permite la elaboración de una serie de tipos sociales atendiendo a la mayor o menor posesión del teléfono en el hogar. Los diez tipos construidos se muestran en el cuadro 2, y sus características en la tabla 6. Al tipo I pertenecen 102 personas, el 4,1 por 100 de la población española, y se caracteriza por sus bajos ingresos y por una escasa cobertura telefónica.

El siguiente grupo con menor posesión de teléfono es el tipo $I V$, formado por los entrevistados con ingresos entre 150.001 y 200.000 pesetas y que se encuentran en paro, y al que pertenece un 6,3 por 100 de la población española. En el tipo II la tasa de posesión del teléfono llega hasta el 73 por 100, y está formado por trabajadores con ingresos mensuales entre 50.001 y 150.000 pesetas. Estos tipos tienen una tasa de cobertura telefónica - al menos- diez puntos inferior al promedio nacional, y representan una quinta parte de la población española. 


\section{CUADRO 2}

Tipos sociales atendiendo a la posesión de teléfono

Tipo I: $\quad$ Ingresos mensuales menores a 50.000 pesetas.

Tipo II: Ingresos mensuales entre 50.001 y 150.000 pesetas, trabajadores.

Tipo III: Ingresos mensuales entre 150.001 y 200.000 pesetas, trabajadores.

Tipo IV: Ingresos mensuales entre 150.001 y 200.000 pesetas, parados.

Tipo V: Ingresos mensuales entre 150.001 y 200.000 pesetas, no población activa.

Tipo VI: Ingresos mensuales superiores a las 200.001 pesetas, trabajadores, hogares formados por 1 ó 2 personas.

Tipo VII: Ingresos mensuales superiores a las 200.001 pesetas, trabajadores, hogares formados por 3 y más personas.

Tipo VIII: Ingresos mensuales superiores a las 200.001 pesetas, parados.

Tipo IX: No responden a la pregunta sobre ingresos mensuales, residen en municipios menores de 2.000 habitantes y mayores de 1.000.001 habitantes.

Tipo X: No responden a la pregunta sobre ingresos mensuales, residen en municipios entre 2.001 y 1.000 .000 de habitantes.

Con una tasa de posesión del teléfono similar a la población total se encuentran los tipos $X, V$ y $I I I$, que representan el 51,2 por $100(17,3+27,1+$ $7,2)$ de la población española.

Por último, la mayor cobertura telefónica se produce en los colectivos de mayores ingresos y cuyos entrevistados se encuentran en paro (tipo VIII), en los colectivos de mayores ingresos que viven en hogares formados por tres y más miembros (tipo VII), así como en las personas que no responden la pregunta sobre el nivel de ingresos y que residen en los municipios más pequeños y mayores (tipo IX). Estos tres subgrupos con alto nivel de cobertura representan el 26 por 100 de la población española.

A modo de conclusión y resumen final de este apartado, señalar que los ingresos mensuales familiares explican la mayor parte de la variación asociada con la cobertura telefónica, seguida por la relación con la actividad del entrevistado. Otras variables que influyen, aunque mucho menos, son el número de personas del hogar y el tamaño del municipio donde se reside. La muestra queda así dividida en diez grupos, con una tasa de cobertura telefónica que oscila entre el 60 y el 97 por 100.

Un análisis similar realizaron Thornberry y Massey (1988: 35-36) a mediados de la década de los ochenta en los Estados Unidos, considerando como variables independientes —además de las incluidas en la tabla 5- la raza del entrevistado y el tipo de empleo. La variable que presentó más influencia fue el nivel de ingresos de la familia, dividido en dos grupos: menores y mayores de $15.000 \$$. El colectivo de menores ingresos resultó dividido por la edad y poste- 
riormente por el nivel educativo, mientras que el colectivo con ingresos superiores se ha segmentado por el nivel de estudios.

\section{TABLA 6}

Características de los tipos sociales ordenados según la tasa de cobertura telefónica

\begin{tabular}{|c|c|c|c|}
\hline Tipo & Tamaño (n) & Tamaño (\%) & Posesión teléfono (\%) \\
\hline I & 102 & 4,1 & 58,8 \\
\hline IV & 157 & 6,3 & 68,8 \\
\hline II & 221 & 8,8 & 72,85 \\
\hline VI & 75 & 3,0 & 80,0 \\
\hline $\mathrm{X}$ & 433 & 17,3 & 81,8 \\
\hline $\mathrm{V}$ & 687 & 27,1 & 83,7 \\
\hline 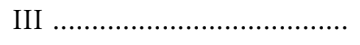 & 180 & 7,2 & 83,9 \\
\hline IX & 139 & 5,6 & 91,4 \\
\hline …........................... & 295 & 11,8 & 92,5 \\
\hline 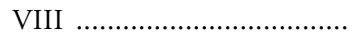 & 211 & 8,4 & 96,7 \\
\hline
\end{tabular}

\section{CONCLUSIONES Y PROPUESTAS DE REDUCCIÓN DEL ERROR DE COBERTURA EN LAS ENCUESTAS TELEFÓNICAS}

Comenzábamos este trabajo planteando la importancia del error de cobertura en las investigaciones que utilizan encuestas telefónicas, preocupados fundamentalmente por los sondeos electorales. La necesidad de recoger rápidamente la información en este tipo de estudios lleva a los investigadores a utilizar la encuesta telefónica en numerosas ocasiones, movidos fundamentalmente por la rapidez en la recogida de información.

Sin embargo, la utilización de este procedimiento puede proporcionar estimaciones de la población que no se ajustan a la realidad, por el hecho que no toda la población dispone de teléfono. A la hora de evaluar este error de cobertura hay que considerar, por una parte, la magnitud de la población no cubierta telefónicamente y, por otro lado, las diferencias entre la población con y sin teléfono,

En los apartados 3 y 4 se ha analizado la magnitud de este problema en la sociedad española, con una tasa de cobertura telefónica de 83 por 100, y con unas importantes diferencias entre los que poseen y no poseen teléfonos (tabla 5). La utilización del análisis de segmentación ha permitido conocer los rasgos asociados a cada "nivel de cobertura», destacando que el nivel de ingresos es la variable que más determina la instalación o no del teléfono en el hogar. En la 
figura 2 se han mostrado los resultados obtenidos mediante el análisis de segmentación. Un análisis de la misma permite comprobar que los ingresos mensuales familiares explican la mayor parte de la variación asociada con la cobertura telefónica, seguida por la relación con la actividad del entrevistado. Una influencia menor presentan el número de personas del hogar y el tamaño del municipio donde se reside. La muestra queda dividida en diez grupos, con una cobertura telefónica que oscila entre el 60 y el 97 por 100 . Un 26 por 100 de la población (subgrupos 7,8 y 9) presenta una cobertura telefónica superior al 90 por 100 , y se caracteriza por tener ingresos superiores a las 200.001 pesetas y vivir en familias de más de dos miembros. Los entrevistados que no responden la pregunta sobre los ingresos y que viven en núcleos poblacionales muy pequeños y muy grandes también forman parte de este colectivo con una elevada cobertura.

El grupo menos cubierto telefónicamente son las personas con ingresos inferiores a las 50.000 pesetas. Con un nivel de cobertura ligeramente superior se encuentran los trabajadores con ingresos entre 50.001 y 150.000 pesetas, y los parados con ingresos mensuales entre 50.000 y 200.000 pesetas. Estos colectivos tienen una tasa de cobertura telefónica —al menos- diez puntos inferior al promedio nacional, y representan una quinta parte de la población española, un 20 por 100 de los entrevistados (subgrupos 1,2 y 3 ).

El hallazgo más importante de este análisis es la localización de la importancia de los ingresos familiares y la relación con la actividad del entrevistado a la hora de explicar la cobertura telefónica, así como el tamaño del municipio de residencia y el tamaño de la familia. No deja de ser sorprendente que otras variables como la comunidad autónoma donde se reside, la edad, el estado civil, el nivel educativo y la ideología no presenten influencia en la presencia del teléfono en el hogar.

Pese a estos problemas, un gran número de investigaciones ignoran el sesgo que puede cometerse en los resultados de una investigación al no considerar los hogares sin teléfono, basados en tres razonamientos: a) en primer lugar, se asume que los hogares sin teléfono tienen un efecto pequeño o despreciable sobre el resultado final; $b$ ) una segunda razón tiene su origen en el elevado coste que supone incluir los hogares sin teléfono; y c) por último, destacan el hecho que la mayor parte de las investigaciones mediante encuestas telefónicas buscan resultados totales, perdiendo importancia el análisis de los subgrupos. Dos de los mayores expertos en la materia, Czaja y Blair, señalan que la mayor parte de las ocasiones estas asunciones son falsas, y la no consideración de estos hogares implica grandes problemas de representatividad (1996: 17).

Llegados a este punto, es el momento de plantear cómo solucionar este problema, presentando diversas estrategias que pueden utilizarse para solucionar la falta de cobertura en las encuestas telefónicas. Azorín y Sánchez Crespo (1992: 46) señalan dos métodos para estimar el error de cobertura, que ellos denominan como el método de re-enumeración y el método de las principales componentes demográficas. 
El primero consiste en volver a enumerar las unidades, en una submuestra de pequeñas áreas, utilizando agentes con una mayor cualificación y experiencia. La ventaja de este método es que permite desvelar la naturaleza del error de cobertura, mientras que entre sus inconvenientes destacan que esta re-enumeración puede producir nuevos errores de cobertura.

El método de las principales componentes demográficas consiste en una comparación entre los datos obtenidos por la investigación con los datos de toda la población. Uno de los problemas es que con este proceso estamos cuantificando no sólo los errores producidos por la falta de cobertura, sino también los generados por otros motivos como el error de medida, los errores voluntarios e involuntarios producidos por el entrevistado, los errores provocados por el entrevistador, la fiabilidad de las preguntas, etc., de modo que es posible obtener grandes diferencias con los valores poblaciones, diferencias que pueden no tener su origen en la falta de cobertura (Groves, 1989: 187).

Otro de los factores que dificultan tremendamente una correcta utilización de esta estrategia es la localización de documentos que reflejen con exactitud las características de la población objeto de estudio, la necesaria actualización de esta información y los escasos datos que suelen incluir estos documentos. Respecto a este último punto, en determinadas ocasiones tan sólo será posible comparar unas pocas variables, generalmente las sociodemográficas, que no permiten conocer adecuadamente la magnitud del problema. En otros momentos nos encontramos con que los documentos que utilizamos para realizar la comparación presentan grandes deficiencias, como ya señalamos en páginas anteriores.

Como la falta de cobertura es uno de los mayores problemas de la encuesta telefónica, se han realizado numerosas investigaciones para evaluar específicamente el impacto de la falta de cobertura con este procedimiento de recogida de información. Una de las estrategias más utilizadas se fundamenta en la elaboración de ponderaciones que compensen, al menos en parte, la omisión de hogares sin teléfono. Para ello, la ponderación requiere utilizar variables conocidas que estén relacionadas con la cobertura. Una vez conocidas las variables que afectan la posesión de teléfono y la distribución de la población objeto de estudio, bastará con asignar una ponderación mayor a los estratos menos representados. Como estas variables suelen estar relacionadas con las variables objeto de estudio, la ponderación conseguirá una mejora de las estimaciones.

Esta ponderación reducirá el sesgo de selección, aunque producirá aumentos en la varianza de las estimaciones, reduciendo con ello la precisión de éstas. Sin embargo, el mayor problema se presenta cuando las variables principales de la investigación tienen una relación débil con las variables utilizadas en la ponderación, o si la forma de esta relación es inusual. En estos casos, la ponderación puede no corregir el error de no cobertura (Massey y Botman, 1988b: 155-158; Smith, 1990: 382-386; Thornberry y Massey, 1988: 45-47).

En estos casos puede que sea conveniente un equilibraje a priori modificando las cuotas de selección de los entrevistados y realizando más entrevistas 
en los colectivos con menor cobertura telefónica (personas de bajos ingresos, etc). La decisión de utilizar o no estos procedimientos varía en función del objeto de estudio y los recursos disponibles. Así, su utilización será imprescindible cuando el objeto de la investigación sean los colectivos con escasa presencia del teléfono, como los señalados en la tabla 5.

\section{REFERENCIAS UTILIZADAS}

Alós, J. S. (1997): «Industria de los Estudios de Mercado en España 1996», Investigación y Marketing, vol. 56, pp. 73-74.

- (1998): «Industria de los Estudios de Mercado en España 1997», Investigación y Marketing, vol. 61, pp. 61-62.

- (1999): «Industria de los Estudios de Mercado en España 1998», Investigación y Marketing, vol. 64, pp. 74-76.

Azorín, F., y SÁnchez Crespo, J. L. (1992): Métodos y aplicaciones del muestreo, Madrid: Alianza.

Bethlehem, J. G., y Kersten, H. M. P. (1985): «On the Treatment of Nonresponse in Sample Surveys», Journal of Official Statistics, vol. 1, pp. 287-300.

Benthlehem, J. G., y KeLleR, W. J. (1989): New technologies in computer assisted survey processing, Vitoria: Euskal Estatistika Erakundea-Insituto Vasco de Estadística, Gobierno Vasco.

BLYTH, B. (1998): «The current and future use of technology in european survey research», conferencia New Methods for Survey Research, 21-22 agosto 1998, Universidad de Southampton, Reino Unido.

Bosch, J. L., y Torrente, D. (1993): Encuestas Telefónicas y por Correo, Madrid: Centro de Investigaciones Sociológicas, colección Cuadernos Metodológicos, núm. 9.

U. S. Bureau of the Census (1975): Course of Nonsampling Errors, International Statistical Program Center, Bureau of the Census, Washington, DC.

Carabaña, J., et al. (1992): Encuesta de Estructura, Conciencia y Biografía de Clase. Informe Técnico, Consejería de Economía, Comunidad de Madrid.

Centro de InVESTIGACIONES SOCIOlÓGICAS (1998): Los comportamientos económicos de los españoles: ahorro y consumo, Madrid: Centro de Investigaciones Sociológicas, estudio núm. 2287, realizado en octubre de 1998.

CIRES, Fundación (1991): Investigación sobre Estilos de Vida, realizada en octubre de 1991 a una muestra representativa de la población española.

CaZjA, R., y Blair, J. (1996): Designing Surveys, California: Pine Forge.

DíAZ DE RADA, V. (2000): «Modos de entrar en relación para la toma de datos: entrevista personal, telefónica y postal», en La Investigación en Marketing, Barcelona: AEDEMO, en prensa.

ESCOBAR, M. (1998): «Las aplicaciones del análisis de segmentación: el procedimiento Chaid», en Empiria, Revista de Metodología de las Ciencias Sociales, núm. 1, pp. 13-50.

Fernández Santana, J. O. (1994): Diseño y utilidad de las encuestas preelectorales, Vitoria: Gobierno Vasco.

FERnÁndeZ Prados, J. F. (1999): «Sistemas informatizados para las encuestas telefónicas», en Metodología de Encuestas, vol. 1, núm. 1, pp. 29-46.

FOWLER, F. J. (1995): Improving Survey Questions, Londres: Sage Publications.

GonZÁlez, J. J. (1998): "Política y demoscopia. Los sondeos y las elecciones generales de 1996», en Empiria, Revista de Metodología de las Ciencias Sociales, núm. 1, pp. 181-202.

Goyder, J. (1985): «Face to Face Interviews and Mailed Questionnaires: the Net Difference in Response Rate", Public Opinion Quarterly, vol. 49, pp. 234-252.

- (1987): The Silent Minority, Cambridge: Polity Press. 
González Gómez, A., y Padilla García, J. L. (1998): «Un esquema conceptual para analizar la validez en las investigaciones mediante encuesta», en Metodología de Encuestas, vol. 1, pp. 85-98.

Groves, R. M.; Biemer, P. P.; Lyberg, L. E.; Massey, J. T.; Nicholls, W. L., y WaksberG, J. (1988): Telephone Survey Methodology, Nueva York: Wiley.

Groves, R. M. (1989): Survey error and survey cost, Nueva York: Wiley.

- (1990): "Theories and Methods of Telephone Surveys», Annual Review of Sociology, vol. 16, pp. 221-240.

Haberman, S. J. (1973): "The Analysis of Residuals in Cross-Classified Tables», Biometrics, vol. 29, pp. 205-220.

InSTITUTO NACIONAL DE EsTADístiCA (INE) (1968): Encuesta de equipamiento y nivel cultural de las Familias, Madrid: INE.

- (1976): Encuesta de equipamiento y nivel cultural de las familias (abril 1975), vol. I, Madrid: INE.

- (1987): Censo de viviendas 1981, tomo 1: Resultados nacionales, Madrid: INE.

- (1992): Encuestas de Presupuestos Familiares 1990/91, Madrid: INE.

Keeter, S. (1995): «Estimating telephone noncoverage bias with a telephone survey», Public Opinion Quarterly, vol. 59, pp. 196-217.

KIsH, L. (1965): Survey Sampling, Nueva York: Wiley. Versión española en 1965: Muestreo de encuestas, Méjico: Trillas.

LATIESA, M. (1997): La enseñanza de la metodología sociológica: estadística, método y técnicas, Armilla: Proyecto Sur de Ediciones.

Lavrakas, P. J. (1993): Telephone Survey Methods, Newbury Park-California: Sage.

- (1996): "To Err is Human», en Marketing Research, vol. 8, pp. 30-36.

LeSSlER, J. T., y KALSBEEK, W. D. (1992): Nonsampling Error in Surveys, Nueva York: Wiley.

MAKLAN, D., y WAKSBERG, J. (1988): "Within-household coverage in RDD surveys», en R. M. Groves et al., Telephone Survey Methodology, Nueva York: Wiley, pp. 51-72.

Manzano Arrondo, V. (1998): "Selección de muestras», en A. J. Rojas, J. S. Fernández y C. Pérez (eds.), Investigar mediante encuestas, Madrid: Síntesis, pp. 51-97.

MARTON, A. (1988): Sampling and non-sampling errors in surveys, Vitoria: Euskal Estatistika Erakundea-Insituto Vasco de Estadística, Gobierno Vasco.

MASSEY, J. T. (1988a): "An overview of telephone coverage», en R. M. Groves et al., Telephone Survey Methodology, Nueva York: Wiley, pp. 3-8.

MASSEY, J. T., y BotTMAn, S. L. (1988b): «Weighting adjustmente for random digit dialed surveys», en R. M. Groves et al., Telephone Survey Methodology, Nueva York: Wiley, pp. 143160.

Miguel, A. de (1993): La Sociedad Española 1992-93, Madrid: Editorial Complutense.

Nicholls, W. L. (1988): «Computer Assisted Telephone Interviewing: a general introduction», en R. M. Groves et al., Telephone Survey Methodology, Nueva York: Wiley, pp. 377-387.

OKSENBERG, L., y CANNEL, C. (1988): «Effects of interviewer vocal characteristics on nonresponse», en R. M. Groves et al., Telephone Survey Methodology, Nueva York: Wiley, pp. 257272.

READER's Digest, Selecciones del (1960): Retrato de dos familias.

Rodríguez Osuna, J. (1991): Métodos de Muestreo, Madrid: Centro de Investigaciones Sociológicas, colección Cuadernos Metodológicos, núm. 1.

SÁNCHEZ-Crespo, J. L. (1973): Muestreo de poblaciones finitas aplicado al diseño de encuestas, Madrid: INE.

SMITH, T. W. (1990): «Phone home? An analysis of househld telephone ownership», International Journal of Public Opinion Research, vol. 2, pp. 369-390.

Steen, C. G. (1983): «Trends in Nonresponse Rates: 1952-1979», Public Opinion Quarterly, vol. 45, pp. 40-57.

SugrYama, M. (1992): "Responses and Non-responses», en Lebart (ed.), Quality of Information in Sample Surveys, París: Dunod, pp. 227-239. 
Thomas, R., y Purdon, S. (1994): «Telephone Methods for Social Surveys», Social Research Update, vol. 8, pp. 1-6.

Thomberry, O. T., y Massey, J. T. (1988): «Trends in United States Telephone Coverage Across Time and Subgroups», en R. M. Groves et al., Telephone Survey Methodology, New York: Wiley, pp. 25-50.

Trasobares, J. L. (2000): “¿... y por qué fallan (o aciertan) las encuestas?», en Heraldo de Aragón, 19 de marzo, p. 5.

TREWIN, D., y LEE, G. (1988): «International comparisons of telephone coverage», en R. M. Groves et al., Telephone Survey Methodology, New York: Wiley, pp. 9-24.

Tucker, C.; Lepkowski, J. M.; Casady, R. J., y Groves, R. M. (1992): «Commercial Residential Telephone Lists: their characteristics and uses in survey design", Social Science Computer Review, vol. 10, pp. 158-172.

Wert, J. I. (1994): «La Encuesta Telefónica», en J. Ibáñez, F. Alvira y M. García Ferrando, El Análisis de la Realidad Social, Madrid: Alianza, pp. 177-188.

- (1996a): «El retorno de los brujos», en El País, 5 de abril, p. 10.

- (1996b): Carta abierta a un incrédulo sobre las encuestas y su muy disputado crédito, Madrid: Península.

\section{ANEXO: FICHA TÉCNICA DE LAS ENCUESTAS DE INTENCIÓN DE VOTO PUBLICADAS EN LA CAMPAÑA ELECTORAL DE LAS ELECCIONES GENERALES DE 2000}

$A B C, 5$ de marzo de 2000.

Realización:

Universo:

Muestra:

Error muestral: $\quad \pm 0,81$ por 100 para un nivel de confianza del 95,5 por 100 y $\mathrm{p}=\mathrm{q}$.

Tipo entrevista: Telefónica asistida por ordenador (CATI).

Trabajo de campo: Del 25 al 29 de febrero.

Diario de Navarra, 4 de marzo de 2000.

Realización:

Universo:

Muestra:

Error muestral:

Tipo entrevista:

Trabajo de campo:

Ipsos-Eco Consulting.

Población española de 18 y más años, residente en todo el territorio nacional.

15.600 entrevistas, distribuidas utilizando un criterio de afijación óptima con un mínimo de 150 entrevistas por circunscripción y un máximo de 600 . 
El Mundo, 5 de marzo de 2000.

Realización:

Sigma-Dos.

Universo:

Personas mayores de 18 años, ámbito nacional.

Muestra: 12.000 entrevistas realizadas mediante un muestreo polietápico, estratificado y aleatorio.

Error muestral: $\quad \pm 0,91$ por 100 para un nivel de confianza del 95,5 por 100 y $\mathrm{p}=\mathrm{q}$.

Tipo entrevista: Telefónica.

Trabajo de campo: Del 21 al 29 de febrero.

El Pais, 5 de marzo de 2000.

Realización:

Universo:

Muestra:

El Periódico de Cataluña, 5 de marzo de 2000.

Realización:

Vox Pública.

Universo:

Muestra: $\quad$ Estratificada por regiones y tamaño del hábitat. Selección

Error muestral: Para un nivel de confianza del 95,5 por 100 y $\mathrm{p}=\mathrm{q}=0,5$ : en

Individuos de 18 y más años residentes en hogares con teléfono en España. afijación uniforme por circunscripción (100 en cada una) y 9.800 con afijación proporcional a la población electoral de cada provincia. La muestra provincial mayor es la de Madrid, con 1.348 entrevistas, y la menor la de Melilla, con 114 .

Error muestral: Considerando un nivel del confianza del 95,5 por 100 , los datos totales presentan un error de $\pm 0,84$ por 100 ( $\mathrm{p}=\mathrm{q})$.

Tipo entrevista: Personal en el domicilio, con selección final del entrevistado mediante el sistema de rutas aleatorias.

Trabajo de campo: Del 25 de febrero al 2 de marzo.

Mayores de 18 años, residentes en la Península, Baleares y Canarias. aleatoria de individuos según cuotas de sexo y edad. Tamaño muestral 2.300 entrevistas, de las que 800 corresponden a Cataluña. Cataluña, $\pm 3,5$ por 100 : en el total de España, $\pm 2,4$ por 100.

Tipo entrevista: Telefónica asistida por ordenador (CATI).

Trabajo de campo: Del 28 de febrero al 1 de marzo. 
La Vanguardia, 5 de marzo de 2000.

Realización:

Instituto Opina.

Muestra:

3.000 entrevistas.

Error muestral: A nivel de confianza del 95 por 100, margen de error del $\pm 1,79$ por 100 .

Tipo entrevista: No consta.

Trabajo de campo: Del 24 al 28 de febrero.

\begin{abstract}
This paper aims to analyse one of the main problems of surveys that are conducted over the telephone, specifically in relation to the process of appraising and learning about those individuals who cannot be interviewed over the telephone because they do not have one: this is defined as coverage error. Despite the "massive» introduction of the telephone into the majority of homes in the developed countries, not every household possesses this means of communication, and there are groups which still lack this piece of equipment. The big problem produced by lack of coverage is the loss of representation which occurs when a particular type of person has to be bypassed, and so this decrease in representativeness becomes a loss of inference ability of the sample, thereby producing a warp in the inference process. Quantification of these groups and knowledge of their characteristics are the main aims of this paper, insofar as they allow us to learn about the magnitude of coverage error in the telephone surveys carried out in Spain.
\end{abstract}

